\title{
When the going gets tougher: the importance of long-term supportive psychotherapy in psychosis
}

\author{
Alan Meaden \& Susie Van Marle
}

\begin{abstract}
There has been considerable progress in recent years in developing psychosocial interventions for people who experience persistent psychotic symptoms. However, it is sometimes difficult to generalise these findings into routine clinical practice. Long-term, psychodynamically informed, supportive psychotherapy is a valuable approach for working with individuals for whom current psychosocial interventions are ineffective or where unhelpful team reactions are obstacles to care. Its principles are used to inform a multiaxial formulation, which is shared with the treatment team and guides treatment, promoting good-quality comprehensive routine care. The benefits of this approach can best be seen at the individual case level using a subsequent multiaxial reformulation.
\end{abstract}

Cognitive-behavioural therapy (CBT) and other psychosocial approaches - such as behavioural family therapy, identifying early warning signs of relapse and developing coping strategies for managing them - have gained steady acceptance as routine best practice interventions in the UK (e.g. Fowler et al, 1995; Birchwood, et al, 2001; National Institute for Clinical Excellence, 2002; Morrison et al, 2004). Given this, it is perhaps surprising that some people with psychosis continue to do badly even when these are offered as early interventions (e.g. Craig et al, 2004). Holloway (2005) has noted that, despite best practice interventions and functionalised community mental health teams (see below), people with severe mental illnesses such as schizophrenia may still experience considerable distress and disability. Furthermore, the long-term success of these interventions has not been clearly demonstrated: there are reports that initial benefits have been lost by 18-month follow-up (e.g. Jones et al, 2004).

It is difficult to define groups or subgroups for whom such interventions are not accepted or not effective in the long term. Kingdom \& Turkington (2005) have proposed an individualised CBT approach for addressing different symptom clusters in schizophrenia. Symptom approaches, however, do not necessarily fully address the broader complex range of psychological needs of people with longterm mental health problems.

For those not able to engage with psychosocial interventions, routine good-quality comprehensive care guided by the care programme approach (CPA) (Department of Health, 1990) is crucial. This process is the cornerstone of mental health practice in England and underpins strong multidisciplinary team working and the development and review of care plans in ensuring high-quality, robust and flexible care. Nevertheless, the CPA may be carried out inconsistently and may be misused (Holloway, 2006). Perhaps more worrying is the fact that routine assessment of patients for purposes of care planning often uncovers high levels of need that continue to be unmet (Marshall et al, 2004).

Adopting a multiaxial framework using the five axes of DSM-IV (American Psychiatric Association, 1994) and drawing on the principles of long-term psychodynamically informed supportive psychotherapy offers one means of promoting good-quality comprehensive routine care for people with the most complex psychiatric difficulties. This approach is particularly relevant for individuals for whom alternative psychosocial interventions have proven ineffective or for those who, for a variety of reasons (see below), cannot engage with more structured

Alan Meaden is a consultant clinical psychologist and psychology lead specialising in psychiatric rehabilitation with Birmingham and Solihull Mental Health NHS Trust (Psychology Services, 208 Monyhull Hall Road, Kings Norton, Birmingham B30 3QJ, UK. Email: alan.meaden@bsmht.nhs.uk). He is also lead for the trust's Psychological Interventions Research Programme and project manager for reviewing residential rehabilitation services. Other clinical and research interests include engagement, working with staff groups, and challenging behaviour and risk. Susanna Van Marle retired from her post as part-time consultant psychiatrist in psychotherapy in 2006. She was involved in applying psychotherapeutic thinking to help patients with severe mental health difficulties and the staff involved in their care. Her clinical and research interests include assessment, consultation, long-term supportive psychotherapy and staff support. 
interventions. The principles of such therapy are especially useful in addressing patient-related dynamics that may prove particularly disruptive to providing good-quality care.

\section{Principles of long-term supportive psychotherapy}

Supportive psychotherapy has been developed as an approach to address the long-term difficulties of patients with chronic diseases and complaints (Fenton, 2000). Its principles have been outlined for working with personality disorders (Van Marle \& Holmes, 2002) and summarised for rehabilitation psychiatry (Davenport, 2006). Particular emphasis is placed on achieving a psychodynamic understanding of the client and their difficulties as well as giving consideration to the more practical tasks of assessment and management. It is extremely important to develop a long-term therapeutic relationship with the client, providing support over different phases of recovery (and relapse) and offering consultancy, support and supervision to others most closely involved in the individual's care.

\section{Therapeutic aims}

The aims of long-term supportive psychotherapy (Box 1) are adapted for each client and interventions are offered flexibly. The longer duration of therapy allows this tailoring to the individual's changing needs, phase of illness and recovery. Therapeutic aims are achieved through the development and sharing of a multiaxial formulation, development of a well-communicating support network or casespecific team (Alanen, 1997) and provision of a frequency and length of therapeutic contact that is sustainable for both the client and the practitioner. Establishing a support network is essential in promoting good continuity and consistency of care. This network comprises those most involved with the client: these are usually members of the multidisciplinary team, but may extend to include others such as the client's general practitioner (GP) and support workers.

\section{Therapeutic techniques}

The techniques of the approach (Box 2) are rehabilitative (Frank, 1982), emphasising adjustment and coping with ongoing difficulties. They are compatible with a recovery philosophy of 'learning how to live, and how to live well, with enduring symptoms and vulnerabilities' (Roberts \& Wolfson, 2006: p. 18).
Box 1 The therapeutic aims of long-term supportive psychotherapy

- Establishment of a therapeutic alliance, taking into account psychological and practical barriers to engagement

- Holding and containing (for the client and the multidisciplinary team)

- Promoting awareness of transference issues (with the client and the team)

- Promoting stability, by improving adjustment and coping as well as reducing relapses and the inappropriate use of services

- Facilitating the maturation of defences and promoting a better adaptation to reality

These therapeutic aims and techniques are not, of course, unique to this approach, and elements are to be found in many psychotherapies and health teams (Margison, 2005) practising good-quality comprehensive routine care. They are, however, strongly advocated by practitioners of long-term supportive psychotherapy. The multiaxial shared formulation framework is the mechanism adopted for ensuring an adequate assessment of needs and promoting a shared understanding of the client's various complex difficulties and how these interact. The therapeutic principles both inform this process and guide how the formulation is translated into routine care planning and management.

Box 2 The therapeutic techniques of longterm supportive psychotherapy

- A style of communication characterised by emphatic validation, praise and advice, and gentle confrontation

- Environmental interventions (e.g. health promotion, enabling access to community resources and support)

- Psychoeducation (including identifying and managing early warning signs of relapse)

- Improving self-awareness (e.g. of defences) and developing coping/adaptive strategies to promote a sense of agency, control and self-management

- A needs-adapted approach: perhaps an emphasis on fostering personal growth, separation and individuation; or a focus on maintenance, preventing deterioration and weathering relapses 


\section{Transference and team holding}

Psychodynamic principles

Awareness of team transference issues may be particularly relevant given the new functionalised community mental health teams (e.g. rehabilitation and recovery, assertive outreach, and primary care liaison teams) being introduced within the National Health Service (Department of Health, 2004). Staff working in such teams are expected to work more intensively with clients and facilitate ordinary activities in an effort to foster engagement and promote stability. Sharing the multiaxial formulation may enable team members to reflect on their feelings about the client and identify potential countertransference issues. As Davenport (2006) notes, clients with psychosis and those with histories of abuse often display interpersonal behaviours and dynamics that are difficult to manage and likely to have a negative influence on engagement. Staff may inadvertently re-enact early patterns of abuse and become enmeshed in unhealthy, destructive interactions (e.g. splitting or projective identification). The multiaxial formulation also serves to educate the team about the client's developmental history and attachment patterns, together with likely maintaining and perpetuating factors. The principle of team holding can be used to help minimise negative interactions and attitudes and reduce the potential for staff burnout. Providing supervision and ongoing support for the team are further important roles.

Transference and countertransference are clearly also important in the client-therapist relationship. The psychodynamic principles of long-term supportive psychotherapy suggest that positive transference be actively nurtured but not interpreted, although the therapist should remain mindful of potential idealisation (Van Marle \& Holmes, 2002). Clients are encouraged to understand the reasons for their unhelpful interactions and to modify them. Attention to transference issues may also provide further insight into the client's interpersonal difficulties. This can be used to contribute towards both the team's and the therapist's understanding of the client's ways of relating and their key vulnerabilities. An important principle is to promote survival despite a complex mixture of often chronic symptoms, frequent relapses, crises and other stressful episodes.

\section{Cognitive-behavioural principles}

Cognitive techniques such as clarifying and challenging dysfunctional assumptions and identifying their triggers are used if they are judged likely to be of benefit. Interventions may also concentrate on the activating events for these beliefs by working on environmental stressors. This may involve encouraging the use of creative activities or social interventions such as increasing contact with voluntary organisations or members of the client's support network.

Improving coping strategies is now a wellestablished component of psychosocial interventions for psychosis. Coping strategy enhancement (Tarrier, et al, 1990) emphasises the refocusing of coping skills in order to remediate symptoms. An important principle of long-term supportive psychotherapy is that ongoing efforts be made to promote coping when the client is best able to make use of them.

Psychoeducation (of the client and the team) is an intrinsic part of developing and progressively sharing the multiaxial formulation and it emphasises understanding of current difficulties in a development context. Broader psychoeducation goals with the client may involve education about diagnostic issues and the bio-psychosocial stress-vulnerability model (Nuechterlein \& Dawson, 1984), learning to minimise stress and prevent and manage relapses.

\section{The client group}

Long-term supportive psychotherapy is most suitable for work with individuals who have highly complex and treatment-resistant psychosis. Service reform has moved many of these people into the community, under the care of assertive outreach or similar teams; others seem almost forgotten, remaining in long-term 24-hour nursed provision. There are broadly three situations in which longterm supportive psychotherapy seems to be most beneficial:

1 where the client has a severe and enduring mental health difficulty (e.g. long-standing bipolar affective disorder or schizophrenia) with the additional presentations listed in Box 3;

2 where there has been severe disruption to personality development, leading to personality characteristics that result in enduring and pervasive patterns of emotions and behaviour that prevent recovery (Box 4);

3 where unhelpful team reactions (such as splitting and projective identification), often as a result of 1 and 2, have become obstacles to engagement and delivering care.

\section{Assessment and formulation}

Taking a thorough and comprehensive personal and psychiatric history, and then identifying realistic goals and areas where work needs to be undertaken and deciding by whom (including the client), is especially important for clients with complex needs and is crucial if teams are to try to prevent enmeshment with services and dependency. 


\section{Box 3 Factors indicating suitability for long- term supportive psychotherapy}

- Poor adherence to treatment (including failure to do 'homework')

- Unwillingness or inability to take responsibility for addressing problems

- Enmeshment in a sick role

- Lack of a consistent problem focus

- Persistent problematic service engagement

Importantly, understanding the developmental context (including attachment patterns) of the client's difficulties and how these interact can determine how assessment findings are best translated into appropriate care plan goals.

A thorough assessment should outline which longterm therapeutic aims and techniques are likely to be most helpful; it should also facilitate supervision and consultation opportunities for the professionals involved (Van Marle \& Holmes, 2002). Discussing with the care team their current approach to management, their frustrations and feelings about working with the client, and their aims and objectives can be a useful means of obtaining further information as well as assessing transference issues.

Formulation is the cornerstone of intervention and provides a framework for therapy, allowing the deployment of integrative techniques and reducing the possibility of therapeutic error. In using the multiaxial framework (Box 5) particular attention should be paid to psychodynamic aspects: maturity of ego defences; attachment styles; and relationship patterns (with family and healthcare staff). This provides a context in which clients and staff can make sense of their multiple problems, giving careful consideration to how the five axes interact. Also important are the client's use of coping strategies and the possibility of developing a supporting network.

The formulation is used to foster communication and promote integrated working between professionals by highlighting respective roles and responsibilities. The multiaxial understanding is shared progressively with those in the supporting network and the client, promoting a deeper understanding of the client's difficulties and helping to minimise unhelpful dynamics.

The use of a guiding multiaxial shared formulation and care informed by long-term supportive psychotherapy with individuals affected by psychosis may be best exemplified in the following fictional case study, which is drawn from our routine clinical work and accurately reflects the themes and issues we regularly encounter.

\section{Box 4 Personality characteristics that prevent recovery}

- Strategies such as those for relating to others, seeking help, receiving care, achieving goals or coping with stress are few and are rigidly applied

- Responses and interpretations of events perpetuate and intensify pre-existing difficulties and needs

- Fragility or a lack of resilience under subjective stress, such that the individual is likely to revert to pathological ways of coping, with poor control over emotions and behaviours

\section{Case study}

Diane is a client in her mid-40s with a 20-year history of bipolar affective disorder. She has had 12 admissions to psychiatric hospital, each lasting on average 9 months (the longest stay was 3 years). Her early treatment consisted largely of out-patient psychiatry with support from a community mental health team. She had been prescribed lithium (with partial adherence) and attempts had been made to monitor her mental state. Diane engaged poorly with services and frequently presented in crisis. She was subsequently referred to an assertive outreach team, in the hope of reducing her hospital admissions and helping her to maintain a more stable period of community functioning and service engagement.

Diane's early life was characterised by strict authoritarian parental attitudes and, as the eldest of three children, she was expected to play a key role in helping to bring up her siblings. Diane went on to train as a nurse but was unable to sustain this career owing to the onset of bipolar disorder following the birth of her daughter. Diane subsequently experienced multiple

\section{Box 5 Multiaxial formulation framework}

Axis I Clinical disorders

Other conditions that may be a focus of clinical attention

Axis II Personality disorders Mental retardation

Axis III General medical conditions

Axis IV Psychosocial and environmental problems (past and present)

Axis V Global assessment of functioning Summary

(After American Psychiatric Association, 1994) 
losses in her life, but her loss of a valued role as a nurse and abandonment by her husband probably had the greatest impact on her mental health, precipitating further relapses.

The assertive outreach team initially found it difficult to manage Diane and attempted to introduce established psychosocial interventions, in accordance with best practice. The team noted that Diane appeared to want to be regarded as a 'special' client, placing ever-increasing demands on team members, who consequently became increasingly frustrated with her. At this stage she was referred for psychological therapy. Initial efforts focused on reviewing any benefits from previous interventions and relationships with team members. From this review it became apparent that Diane tended to reject responsibility for change and to locate the source of her difficulties in others, stating that they did not do enough for her, neglected her or caused her unnecessary stress. At this point a multiaxial formulation was developed (Box 6) and her care and management were reviewed.
Integrated working The multiaxial formulation was progressively shared with members of the assertive outreach team, with two main consequences for the team's understanding and approach to treatment. First, it promoted a deeper understanding of Diane's Axis II difficulties, enabling team members to stand back from their interactions and recognise important transference issues and potential therapeutic traps. Second, it helped them to identify more realistic therapeutic aims.

Therapeutic aims and interventions It was clear from the formulation that Diane was likely to require ongoing help, advice and support rather than further attempts to promote change through time-limited manualised interventions. Therapeutic efforts could now be refocused on achieving the level of support necessary to reduce relapses while minimising stress for the support network members (i.e. the members of the assertive outreach team most consistently involved with her care). This meant increasing and maintaining a level of support for Diane that, at a surface level at

\section{Box 6 Multiaxial formulation (July 2003)}

Axis I Diagnostic category

- Bipolar affective disorder (type I) - first admission 1984

- Social anxiety

Axis II Personality disorders and developmental factors

- Narcissistic and dependent personality traits

- Attachment style: insecure - predominantly anxious/enmeshed

- Defences: projection; denial; omnipotence; splitting, idealisation - denigration, passive-aggressive trait; somatisation

Axis III Physical and constitutional factors:

- History of high blood pressure

Axis IV Environmental factors (past and present):

- Unemployment - loss of career as a nurse (1986)

- Husband's affair and abandonment (1987) - lived alone ever since

- Lack of a meaningful role in family

- Mother died 1995 (history of depression)

- Conflict with brother (main carer)

- Limited range of meaningful activities, roles and goals

Axis V Current level of functioning

- Three admissions in past 3 years (average of 6 months duration)

- Poor social functioning

- Not working

- Manic symptoms in response to psychosocial stressors

- Difficulty with interpersonal relationships: with family, friends and professionals

- Psychosocial stressors provoke manic symptoms

Summary - Strict upbringing - emotionally invalidating environment

- Significant and multiple losses - vulnerability to anniversaries of these

- Interpersonal conflict; especially with family members

- Need to be treated as special

- Insecure attachment pattern: predominantly anxious/enmeshed

- Reliance on immature defences

- Family history of depression 
least, appeared to be unnecessary. The therapeutic focus was one of containment and holding, helping Diane to tolerate painful experiences surrounding the multiple losses in her life that frequently surfaced around anniversaries and led to poor emotional coping, demands of reassurance from others, increased physical complaints and escalating manic symptoms (often resulting in hospitalisation).

In light of Diane's tendency to experience others as critical, controlling and rejecting, it was agreed that therapeutic and care interactions should be characterised by offering praise and valuing any efforts (however small) that she made to tackle her difficulties. The care staff used gentle confrontation when she 'catastrophised' everyday problems, pointing out that she had dealt successfully with many similar situations and that help was available to deal with problems if needed. This aimed to promote a positive transference between Diane and team. It also allowed the gradual introduction of new coping skills as well as enhancement of existing ones.

Before the multiaxial formulation, work with Diane had focused on identifying her relapse signature and a drill that she could implement during self-monitoring (Birchwood et al, 2000). However, when subsequent relapses occurred Diane did not report an increase in early warning signs or instigate any features of her relapse drill. Early-signs monitoring was therefore implemented, in which team members monitored her behaviour for indications of relapse (Birchwood et al, 1989). This communicated to Diane that the team was taking her seriously by carefully monitoring her mental state. Early-signs monitoring also allowed the team to increase medication to manage potential early warning signs. This approach increased Diane's self-confidence and her faith in the team. It also created more stable periods when coping strategies could be encouraged and introduced at a pace appropriate to her.
Education and social skills teaching was provided to help Diane to ask for help directly, rather than present with increased somatic concerns. During better periods the importance of pacing daily activities was introduced, with the aim of reducing the risk of precipitating a manic high. Emphasis was also placed on maintaining a daily routine and structure, especially during phases of low mood. The long-term nature of the work allowed for this advice to be reiterated whenever necessary, and the early-signs monitoring gradually encouraged Diane to recognise for herself changes in her mood and activity.

The benefits of our approach for Diane were evident in the reformulation conducted about 3 years later (Box 7).

\section{Efficacy}

It is difficult to disentangle the potential benefits of the approach taken with Diane from other interventions offered in a complex and often dispersed care system. It is a relatively long-term, flexible, non-manualised way of working that does not lend itself readily to trial-based evaluations. Therapeutic outcomes for clients with chronic complex difficulties are likely to be less to do with symptomatic recovery and more focused on areas such as improved use of coping strategies, reductions in periods of crisis, fewer relapses and improved quality of life. These should be the focus of evaluation efforts and form part of the routine evaluation of clinical practice. Such a practice-based evidence approach is particularly relevant given the lack of generalisability of current research findings to those most difficult to treat.

\section{Box 7 Multiaxial reformulation (March, 2006)}

In the reformulation, Axes I-III remained unchanged, as did most of the summary points (see Box 6). Changes on Axes IV and V, and in the summary, are indicated below in italic
Axis IV
Environmental factors (past and present)
- Multiple losses
- Developed a meaningful role in the local community
- Lack of a meaningful role in family
- Conflict with brother
- Involvement in local activities
Axis $\mathrm{V} \quad$ Current level of functioning
- No admissions since March 2003
- Generally good social functioning
- Not working
- Transient symptoms in response to psychosocial stressors
- Able to sustain some interpersonal relationships and limited meaningful relationships
Summary - Significant and multiple losses-increased resilience and coping around their anniversaries 


\section{Conclusions}

Adopting the principles of long-term supportive psychotherapy involving multiaxial shared formulations can be a valuable means of enabling individuals with the most complex and treatment-resistant problems associated with psychosis to learn how to live, and to live well, with enduring symptoms and vulnerabilities. This long-term, team-based approach promotes thorough assessment and comprehensive care. It thus fits well with emerging service models such as assertive outreach and may be better able to promote adjustment, stability and maintenance of therapeutic benefits over the longer term.

\section{Declaration of interest}

None.

\section{References}

Alanen, Y. O. (1997) Schizophrenia: Its Origins and Need-Adapted Treatment. Karnac.

American Psychiatric Association (1994) Diagnostic and Statistical Manual of Mental Disorders (4th edn) (DSM-IV). APA.

Birchwood, M. J., Smith, J., Macmillan, F., et al (1989) Predicting relapse in schizophrenia: the development and implementation of an early signs monitoring system using patients and families as observers. Psychological Medicine, 19, 649-656.

Birchwood, M., Spencer, E. \& McGovern, D. (2000) Schizophrenia: early warning signs. Advances in Psychiatric Treatment, 6, 93-101.

Birchwood, M. J., Fowler, J. \& Jackson, C. (2001) Early Intervention in Psychosis: A Guide to Concepts, Evidence and Interventions. John Wiley \& Sons.

Craig, T. K. J., Garety, P., Power, P., et al (2004) The Lambeth Early Onset (LEO) team: randomised controlled trial of the effectiveness of specialised care for early psychosis. BMJ, 329, 1067-1070.

Davenport, S. (2006) Psychodynamic considerations in rehabilitation. In Enabling Recovery: The Principles and Practice of Rehabilitation Psychiatry (eds G. Roberts, S. Davenport, F. Holloway, et al), pp. 187-199. Gaskell.

Department of Health (1990) The Care Programme Approach for People with a Mental Illness Referred to Specialist Psychiatric Services (HC(90)23). Department of Health.

Department of Health (2004) The National Service Framework for Mental Health - Five Years On. Department of Health.

Fenton, W. S. (2000) Evolving perspectives on individual psychotherapy for schizophrenia. Schizophrenia Bulletin, 26, 47-72.

Fowler, D., Garety, P. \& Kuipers, L. (1995) Cognitive Behaviour Therapy for Psychosis: Theory and Practice. John Wiley \& Sons.

Frank, J. D. (1982) Therapeutic Components Shared by All Psychotherapies. American Psychological Association.

Holloway, F. (2005) The Forgotten Need for Rehabilitation in Contemporary Mental Health Services. A Position Statement from the Executive Committee of the Faculty of Rehabilitation and Social Psychiatry, Royal College of Psychiatrists. Royal College of Psychiatrists. http://www.rcpsych.ac.uk/pdf/frankholloway_oct05.pdf

Holloway, F. (2006) Pulling it all together: the care programme approach at its best. In Enabling Recovery: The Principles and Practice of Rehabilitation Psychiatry (eds G. Roberts, S. Davenport, F. Holloway, et al), pp. 231-242. Gaskell.

Jones, C., Cormac, I., Silveira da Mota Neto, J. I., et al (2004) Cognitive behaviour therapy for schizophrenia. Cochrane Database of Systematic Reviews, issue 4. (CD000524, doi: 10.1002/14651858. CD000524. Wiley InterScience.

Kingdom, D. G. \& Turkington, D. (2005) Cognitive Therapy of Schizophrenia: Guides to Individualised Treatment. Guilford Press.
Margison, F. (2005) Integrating approaches to psychotherapy in psychosis. Australian and New Zealand Journal of Psychiatry, 39, 972-981.

Marshall, M., Lockwood, A., Green, G., et al (2004) Systematic assessments of need and care planning in severe mental illness. Cluster randomised controlled trial. British Journal of Psychiatry, 185, 163-168.

Morrison, A. P., Renton, J. C., Dunn, H., et al (2004) Cognitive Therapy for Psychosis: A Formulation-based Approach. Routledge.

Nuechterlein, K. H. \& Dawson, M. E. (1984) A heuristic vulnerability/stress model of schizophrenic episodes. Schizophrenia Bulletin, 10, 300-312.

National Institute for Clinical Excellence (2002) Schizophrenia: Core Interventions in the Treatment and Management of Schizophrenia in Primary and Secondary Care. Clinical Guideline 1. NICE.

Roberts, G. \& Wolfson, P. (2006) New directions in rehabilitation: learning from the recovery movement. In Enabling Recovery: The Principles and Practice of Rehabilitation Psychiatry (eds G. Roberts, S. Davenport, F. Holloway, et al), pp. 18-37. Gaskell.

Tarrier, N., Harwood, S., Yusupoff, L., et al (1990) Coping strategy enhancement (CSE): a method of treating residual schizophrenic symptoms. Behavioural Psychotherapy, 18, 283-293.

Van Marle, S. \& Holmes, J. (2002) Supportive psychotherapy as an integrative psychotherapy. In Integration in Psychotherapy: Models and Methods (eds J. Holmes \& A. Bateman), pp. 175-195). Oxford University Press.

\section{MCQs}

1 The following principles of long-term supportive psychotherapy are not relevant in the management of complex treatment-resistant psychosis:

a engaging in long-term efforts to promote coping

b achieving a psychodynamically informed understanding

c discouraging positive transference

d promoting awareness of transference issues in the team

e establishing a long-term therapeutic relationship.

2 Multiaxial formulations informed by long-term supportive psychotherapy:

a predict which interventions will be most effective

$\mathrm{b}$ are based on a standardised assessment process

c show how complex problems interact

d make diagnosis unnecessary

e explain previous intervention failures.

3 Principles of long-term supportive psychotherapy best apply to:

a patients with first-episode illness

b patients with complex and treatment-resistant psychosis

c only patients with bipolar disorder

d patients who are recovered

e patients without additional personality difficulties.

4 Patient characteristics and behaviours that most limit recovery include:

a unwillingness or inability to take responsibility for addressing problems

b persistent psychotic symptoms

c a history of frequent relapses

d persistent non-psychotic symptoms

e a history of failed attempts to benefit from services. 
5 The efficacy of multiaxial formulations and care planning informed by long-term supportive psychotherapy are best demonstrated by:
a symptomatic recovery
b reduced team contacts
c longer hospital admissions
d reductions in crises and relapses
e reduced frequency of care programme approach reviews.

\begin{tabular}{ccccccc}
\multicolumn{2}{c}{ MCQ answers } & & & & \\
1 & & 2 & 3 & 4 & 5 & \\
a F & a F & a F & a T & a F \\
b F & b F & b T & b F & b F \\
c T & c T & c F & c F & c F \\
d F & d F & d F & d F & d T \\
e F & e F & e F & e F & e F
\end{tabular}

